\title{
Doenças infecciosas como causas de morte em idosos autopsiados
}

\author{
Infeccious diseases as causes of death \\ in autopsied elderly
}

\author{
Flávia Aparecida de Oliveira ', Marlene Antônia dos Reis ${ }^{1}$, Eumênia Costa da Cunha Castro ', \\ Selma Freire de Carvalho da Cunha $\quad{ }^{2}$ e Vicente de Paula Antunes Teixeira ${ }^{1}$
}

\section{RESUMO}

O envelhecimento populacional no Brasil está associado às alterações na morbimortalidade da população. Nesse estudo, foi verificado as causas de morte e outros processos patológicos em idosos autopsiados. Os idosos com idade maior ou igual a 60 anos, no período de 1976 a 1998 representaram 394 casos (24,4\%). A mediana da idade foi 69 (60 a 120) anos, sendo maior nas mulheres (70,5 versus 68 anos; $p<0,05)$. 0 sexo masculino (67,5\%), a cor branca $(69,8 \%)$ e as causas de morte cardiovascular (43,7\%) e infecciosa (31\%) prevaleceram. A subnutrição (76,2\%) estava associada à pneumonite e cistite, $(p<0,05)$. A arteriosclerose $(61,9 \%)$ e as cardiopatias chagásica (42,1\%) e hipertensiva (39,1\%) foram os processos mais freqüentes. Portanto, foi observado sobreposição das causas de morte crônico-degenerativas e infecciosas. Isso demonstra a necessidade de valorizar medidas como o acompanhamento do indice de massa corporal e dos fatores de risco para o desenvolvimento de doenças no envelhecimento, como a pneumonite.

Palavras-chaves : Autópsia. Idoso. Morbidade. Mortalidade. Processos patológicos.

\section{ABSTRACT}

The population aging in Brazil was associated with the alterations in the morbimortality of the population. The aim of this study was to verify the causes of death and other pathological processes in autopsied elderly. The elderly with 60 years or more in the period from 1976 to 1998 represented 394 cases (24.4\%). The age median was 69 years ranged from 60 to 120 years, being higher in the women (70.5 versus 68yrs; $p<0.05)$. The male gender (67.5\%), white color (69.8\%), and the cardiovascular (43.7\%) and infectious (31\%) causes of death were dominate. In the malnourished (76.2\%) it had association of pneumonia and cystitis, $(p<0.05)$. Arteriosclerosis (61.9\%), and the chagasic (42.1\%) and hypertensive cardiopathies (39.1\%) were the most frequent processes. Therefore, it was observed an overlapping of chronic-degenerative and infectious causes of death. This study shows the necessity to value of follow up of body mass index and of risk factors for the development of illness in aging patients like pneumonia.

Key-words: Autopsy. Elderly. Morbidity. Mortality. Pathologic process.

0 processo de transição demográfica caracterizado pela diminuição das taxas de mortalidade e de fecundidade tem proporcionado o aumento da população idosa mundial. Nos países desenvolvidos, ele ocorreu gradativamente, facilitando a adaptação às condições necessárias para assistir aos idosos ${ }^{2}$. No Brasil, esse processo intensificou-se a partir da década de 60 , e a expectativa de vida ao nascer atingiu 68,6 anos em 2000. 0 processo de envelhecimento no Brasil está associado em geral, com as melhorias das condições médico-sanitárias, enquanto nos países desenvolvidos o processo está associado tanto com a evolução médico-sanitária como com a melhoria das condições de educação, saneamento básico, infra-estrutura, condições sócio-econômicas e outros fatores. Dessa forma, o Brasil apresenta sua população envelhecendo em curto prazo o que dificultou que as modificações para a atenção aos

1. Disciplina de Patologia Geral da Faculdade de Medicina do Triângulo Mineiro, Uberaba, MG. Disciplina de Nutrologia da Faculdade de Medicina do Triângulo Mineiro, Uberaba, MG. Apoio financeiro: Fundação de Ensino e Pesquisa de Uberaba (FUNEPU), Fundação de Amparo à Pesquisa do Estado de Minas Gerais (FAPEMIG) e Conselho Nacional de Desenvolvimento Científico e Tecnológico (CNPq)

Endereço para correspondência: Drª Marlene Antônia dos Reis. Disciplina de Patologia Geral/FMTM. Rua Frei Paulino 30, $38025-180$ Uberaba, MG.

Tel: 34 3318-5428, Fax 343318-5846.

e-mail: mareis.pat@dcb.fmtm.br.

Recebido para publicação em 2/8/2002

Aceito em 13/11/2003 
idosos estivessem estabelecidas paralelamente ao envelhecimento populacional $^{2714}$.

0 envelhecimento populacional favoreceu o processo de transição epidemiológica, caracterizado pela diminuição da incidência das doenças infecto-parasitárias e aumento das doenças crônico-degenerativas. Entretanto, no Brasil, pode ser evidenciada uma sobreposição das duas categorias de enfermidades, mostrando o surgimento de uma nova demanda nas instituições de saúde em busca de prevenção e tratamento 5 . 0 conhecimento da morbidade e das causas de morte nos idosos é de fundamental importância para o planejamento de estratégias visando a promoção e a recuperação da saúde e a profilaxia de doenças ${ }^{2}$. 0 objetivo do presente estudo foi verificar as causas de morte e outros processos patológicos em idosos autopsiados.

\section{CASUÍSTICA E MÉTODOS}

Os laudos de autópsias selecionados para o estudo foram de idosos com 60 anos de idade ou mais, atendidos no Hospital Escola da Faculdade de Medicina do Triângulo Mineiro (FMTM), UberabaMG, no período de 1976 a 1998.

Os dados registrados foram a idade, o sexo, a cor e os dados anatomopatológicos. Estes foram agrupados em causas de morte e outros processos patológicos. As causas de morte foram agrupadas em cardiovascular, infecciosa, neoplásica, digestiva e outras, usando critérios ecléticos ${ }^{13}$. A desnutrição no idoso ${ }^{9}$ foi definida através do cálculo do índice de massa corporal, IMC = Peso em kg/Altura em $\mathrm{m}^{2}$, sendo considerado subnutrição quando $\mathrm{IMC}<22 \mathrm{~kg} / \mathrm{m}^{2} \mathrm{e}$ sobrepeso quando IMC $>27 \mathrm{~kg} / \mathrm{m}^{2}$.

As variáveis qualitativas foram expressas em freqüência e a associação entre elas foi verificada pelo teste do $\chi^{2}$. As variáveis quantitativas que apresentaram distribuição não normal foram expressas em mediana e valores mínimo e máximo, sendo comparadas pelo teste de Mann-Whitney (T). Os resultados foram considerados significativos quando $\mathrm{p}<0,05$. 0 presente estudo foi aprovado pelo Comitê de Ética em Pesquisa (CEP) da FMTM.

\section{RESULTADOS}

De 1612 pessoas autopsiadas no período de 1976 a 1998, 394 casos $(24,4 \%)$ eram de idosos. A mediana da idade foi 69 (60 a 120) anos e a faixa etária mais freqüente foi a de 60 a 69 anos (52,8\%) enquanto que acima de 80 anos a taxa do exame necroscópico foi de 11,9\% (Tabela 1). A mediana da idade das mulheres (70,5 anos) foi significativamente maior que a dos homens (68 anos) $(\mathrm{T}=27.805 \mathrm{p}=0,017)$.

A causa de morte mais freqüente foi a cardiovascular (43,7\%) seguida pela infecciosa (31\%) (Tabela 2). As causas de morte cardiovasculares estavam relacionadas à cardiopatia chagásica em alguns casos e as infecciosas à pneumonite na maioria dos casos. 0 IMC determinado em 265 casos apresentou mediana de 18,8 (10,7 a 38,4$) \mathrm{kg} / \mathrm{m}^{2}$. A pneumonite e a cistite foram significativamente mais freqüentes nos indivíduos subnutridos que nos não subnutridos (Figura 1). Os processos crônicos como enfisema pulmonar, bronquite, cardiopatia chagásica e neoplasia foram mais freqüentes entre os subnutridos, porém a diferença não foi estatisticamente significativa.

As cardiopatias foram encontradas em $71,1 \%$ dos idosos, sendo distribuídas em chagásica (42,1\%), hipertensiva $(39,1 \%)$, isquêmica $(9,1 \%)$ e reumática (2\%), sendo que alguns indivíduos apresentaram mais de uma cardiopatia. Os demais processos patológicos estão apresentados na Tabela 3 .

Tabela 1- Distribuição da faixa etária, do gênero, da cor e do índice de massa corporal (IMC) em idosos autopsiados no Hospital Escola da Faculdade de Medicina do Triângulo Mineiro, Uberaba-MG, no período de 1976 a 1998.

\begin{tabular}{lrr}
\hline Faixa etária (anos) & $\mathrm{n}^{0}$ & $\%$ \\
\hline 60 a 69 & 208 & 52,8 \\
70 a 79 & 139 & 35,3 \\
80 a 89 & 37 & 9,4 \\
$\geq 90$ s & 10 & 2,5 \\
Sexo & & \\
$\quad$ masculino & 266 & 67,5 \\
$\quad$ feminino & 128 & 32,5 \\
Cor & & \\
$\quad$ branco & 275 & 69,8 \\
$\quad$ não branco & 119 & 30,2 \\
IMC $\left(\mathrm{kg} / \mathrm{m}^{2}\right)^{*}$ & & \\
$\quad<22$ & 202 & 76,2 \\
22 a 27 & 49 & 18,5 \\
$\quad>27$ & 14 & 5,3 \\
\hline
\end{tabular}

Total de casos analisados $=394 ; * 0$ índice de massa corporal (IMC) foi calculado em 265 casos.

Tabela 2 - Distribuição da causa de morte em idosos autopsiados no Hospital Escola da Faculdade de Medicina do Triângulo Mineiro, Uberaba-MG, no período de 1976 a 1998.

\begin{tabular}{lrr}
\hline Causa de morte & $\mathrm{n}^{0}$ & $\%$ \\
\hline Cardiovascular & 172 & 43,7 \\
Infecciosa & 122 & 31,0 \\
Neoplásica & 56 & 13,2 \\
Digestiva & 22 & 5,6 \\
Outras & 26 & 6,6 \\
\hline Total & 394 & 100,0 \\
\hline
\end{tabular}

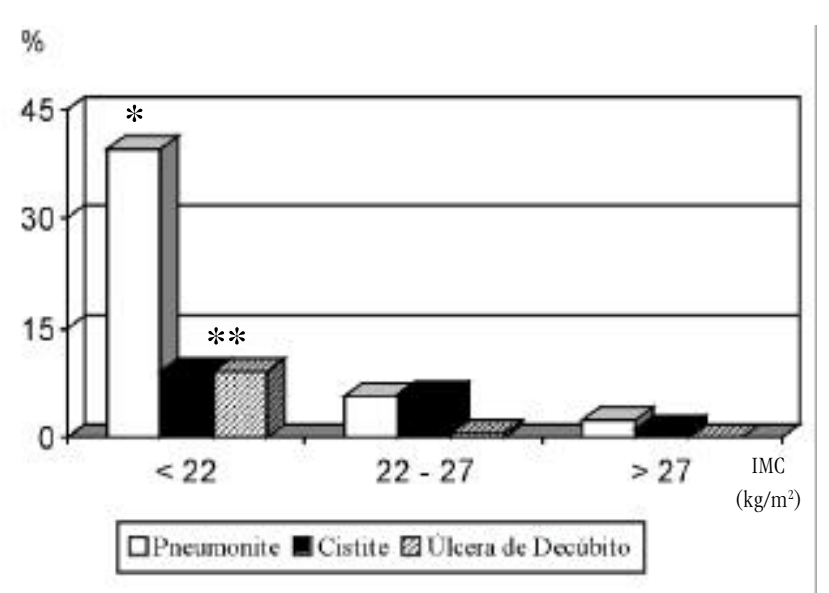

*Subnutridos x pneumonite: $\chi^{2}=5,968 \mathrm{p}=0,015$. **Subnutridos $\mathrm{x}$ cistite: $\chi^{2}=10,496 \mathrm{p}=0,001$. Subnutridos $\mathrm{x}$ úlcera de decúbito: $\chi^{2}=3,189 \mathrm{p}=0,074$.

Figura 1 - Associação entre o indice de massa corporal (IMC) e a pneumonite, a cistite e a úlcera de decúbito em 265 idosos autopsiados no Hospital Escola da Faculdade de Medicina do Triângulo Mineiro, Uberaba-MG, no período de 1976 a 1998. 
Tabela 3 - Distribuição dos processos patológicos em idosos autopsiados no Hospital Escola da Faculdade de Medicina do Triângulo Mineiro, Uberaba-MG, no período de 1976 a 1998.

\begin{tabular}{lrr}
\hline Processos Patológicos & número & porcentagem \\
\hline Cardiopatias & 280 & 71,1 \\
Arteriosclerose & 244 & 61,9 \\
Hemorragia e/ou enfarte cerebrais & 62 & 15,7 \\
Pneumonite & 160 & 40,6 \\
Bronquite & 38 & 9,6 \\
Enfisema & 81 & 20,6 \\
Neoplasia & 81 & 20,6 \\
Úlcera de decúbito & 29 & 7,4 \\
Cistite & 55 & 14,0
\end{tabular}

0 total ultrapassa os 394 (100\%) porque algumas pessoas apresentaram mais de um processo patológico.

A cistite foi mais freqüente no sexo feminino (18\%) que no masculino (12\%), mas não houve diferença estatística. Alguns indivíduos apresentaram múltiplos processos patológicos, 13,7\% dos casos tinham cardiopatias chagásica e hipertensiva. Além dessas cardiopatias, 3\% desses indivíduos tinham também arteriosclerose, hemorragia e/ou enfarte cerebrais, subnutrição e pneumonite.

\section{DISCUSSÃO}

Os estudos referentes à morbimortalidade em idosos autopsiados parecem ser pouco freqüentes no Brasil ${ }^{812}$. No presente estudo, foi verificado nos idosos autopsiados o predomínio das causas de morte cardiovasculares seguida pelas infecciosas que na maioria dos casos estavam relacionadas a pneumonite. Além disso, a pneumonite e a cistite estavam associadas à subnutrição na maioria dos casos.

0 número de autópsias realizadas em idosos foi inversamente proporcional ao aumento da faixa etária, semelhante a outros estudos $^{1}$. Esse fenômeno, provavelmente está relacionado a melhor aceitação da morte e à dificuldade de obtenção do consentimento da família para a realização de autópsias nos idosos ${ }^{1}$. A autópsia é uma fonte rica de informações que permite o levantamento de dados antropométricos e nosológicos que muitas vezes se estabeleceram ao longo da vida do indivíduo e passaram sem registro. Dentre as várias aplicações desses dados, se destaca 0 desenvolvimento educacional dos profissionais de saúde e o controle da qualidade da assistência prestada aos pacientes ${ }^{12} 15$. Além disso, as autópsias são úteis para a compreensão clínica e epidemiológica das morbidades, possibilitando as pesquisas relacionadas aos fatores de risco, ao planejamento dos recursos econômicos e a diminuição da subestimativa clínica de doenças potencialmente tratáveis ${ }^{1215}$.

No presente trabalho, as causas de morte mais freqüentes foram as cardiovasculares $(43,7 \%)$ e as infecciosas (31\%) semelhante aos achados de outros autores que descreveram taxas variando de 32,0 a 54,5\% para as causas de morte cardiovasculares e 36 a 54,6\% para as causas infecciosas ${ }^{4612}$. As causas de morte cardiovasculares estavam relacionada a cardiopatia chagásica em alguns casos semelhante ao verificado em outro estudo ${ }^{8}$. A autópsia no idoso é importante para a definição da causa de morte, pois nas declarações de óbito é freqüente o registro de causas mal definidas nesses indivíduos. No Chile em 1983, as três primeiras causas de morte no idoso, obtidas por meio da declaração de óbito, foram cardiovascular, neoplásicas e doenças mal definidas ${ }^{10}$. Embora as causas de morte crônico-degenerativas tenham sido as mais freqüentes no presente estudo, a freqüência das causas infecciosas nos idosos foi alta, sendo observado uma sobreposição das duas categorias de doenças. As doenças crônico-degenerativas podem ser resultantes em parte, de fatores de risco à saúde acumulados ao longo dos anos, por exemplo: fumo, sedentarismo, álcool, alimentação inadequada e uso de medicamentos 5 .

A alta freqüência da cardiopatia chagásica no presente estudo pode estar associada a endemicidade da doença na região, que pode atingir até 38,8\% em autópsias ${ }^{11}$. A cardiopatia hipertensiva além de interferir na qualidade de vida dos idosos, pode se apresentar como agravante das doenças de base ou como causa de morte ${ }^{16}$. Na Nigéria, em um estudo relacionado à morte súbita em autópsias, todos os idosos morreram por causas cardiovasculares, sendo 77,8\% dos casos por cardiopatias ${ }^{16}$.

A maioria dos idosos no presente estudo pode ser considerada subnutrida. A subnutrição freqüentemente está associada às doenças crônico-degenerativas, que podem levar à anorexia e ao aumento da demanda de nutrientes ${ }^{17}$. Além disso, pode favorecer complicações como a redução da imunidade, o aumento de quedas, a cicatrização precária, a anemia, o edema, a fraqueza muscular e a fadiga ${ }^{5}$. Esses fatores podem propiciar o desenvolvimento de doenças como a pneumonite e a úlcera de decúbito que foram mais freqüentes entre os subnutridos no presente estudo.

A pneumonite foi encontrada em 40,6\% dos casos, sendo na maioria deles relacionada à causa de morte, semelhante a outros estudos que evidenciaram taxas variando de $42,9 \%$ a $59 \%{ }^{456}$. Essa doença pode estar relacionada a fatores de risco como cardiopatias, doença pulmonar obstrutiva-crônica e subnutriçã $0^{3}$, esses processos foram observados em vários indivíduos no presente estudo. A cistite foi mais freqüente entre as mulheres e nos subnutridos. Após a menopausa ocorre o aumento do $\mathrm{pH}$ vaginal que favorece a colonização por gram negativos, além disso, a contaminação perineal devido à incontinência fecal, o uso de cateterismo vesical no ambiente hospitalar e a subnutrição são outros fatores de risco para essa entidade 3 . A presença de múltiplos processos patológicos nos idosos foi freqüente, semelhante a outros estudos ${ }^{412}$. Isso pode determinar a subnotificação de processos potencialmente tratáveis durante a assistência clínica, justificando a realização de autópsias ${ }^{12}$.

Portanto, foi observado sobreposição das causas de morte crônico-degenerativas e infecciosas. A alta freqüência das causas infecciosas e de entidades como a subnutrição e a pneumonite demonstram a necessidade de valorizar medidas como 0 acompanhamento do IMC e a verificação dos fatores de risco para o desenvolvimento de doenças no envelhecimento, como a pneumonite.

\section{AGRADECIMENTOS}

Os autores agradecem a Aloísio Costa, Lourimar J. Morais, Maria Aparecida S. Dias, Maria Helena S.C. Batista e Sônia M. Sobrinho pelas contribuições na realização desse trabalho. 


\section{REFERÊNCIAS BIBLIOGRÁFICAS}

1. Ahroheim JC, Bernholc AS, Clark WD. Age trends in autopsy rates striking decline in late life. Journal of the American Medical Association 250:1182-1186, 1983.

2. Chaimowicz F. A saúde dos idosos brasileiros às vésperas do século XXI: problemas, projeções e alternativas. Revista de Saúde Pública 31:184-200, 1997.

3. Fraser, D. Assessing the elderly for infections. Journal of Gerontology Nurse 23:510, 1997.

4. Gee Mac W. Causes of death in a hospitalized geriatric population: an autopsy study of 3000 patients. Virchows Archiv A Pathological Anatomy and Histopathology 423:343-349, 1993.

5. Guimarães RM. Prevenção das doenças associadas ao envelhecimento. Arquivos de Geriatria e Gerontologia p. 9-14, 1996.

6. Haleem MA. Bronchopneumonia as a cause of death in the elderly. International Journal of Experimental and Clinical Gerontology 28:203-207, 1982.

7. Instituto Brasileiro de Geografia e Estatística. http//: ww.ibge.gov.br/home/estastistica/ populacao/tabuadevida/evolucao_da_mortalidade.shtm.; Acesso em 22/11/2002.

8. Menezes M, Rocha A, Silva AC, Silva AM. Basic causes of death in elderly patients with Chagas' disease. Arquivos Brasileiros de Cardiologia 52:75-78, 1989.

9. Saltzman E, Mason JB. Enteral nutrition en the elderly. In: Rombeau JL, Rolandelli RH (eds) Clinical Nutricional. Enteral and tube feeding. Assessment by anthropometric and biomedical methods. $3^{\text {th }}$ edition WB. Saunders Company, Philadelphia, p. 385-402, 1997.

10. Kirschbaum A, Arias EC. Mortalidad del senescente en Chile. Cuadernos Medico Sociales 29:134-138, 1988.

11. Lopes ER, Chapadeiro E. Doença de Chagas no Triângulo Mineiro. Revista Goiana de Medicina 32:109-113, 1986.

12. Martins EML, Camara VD, Carneiro IR. A importância da realização de necropsias em indivíduos idosos. Arquivos de Geriatria e Gerontologia 2:83-89, 1998.

13. Reis MA, Costa RS, Ferraz AS. Causes of death in renal transplant recipients: a study of 102 autopsies from 1968 to 1991. Journal of the Royal Society of Medicine 88:24-27, 1995.

14. Ramos LR, Veras RP, Kalache A. Envelhecimento populacional: uma realidade brasileira. Revista de Saúde Pública 21:211-224, 1987.

15. Roosen J, Frans E, Wilmer A, Knockaert DC, Bobbaers H. Comparison of premortem clinical diagnoses in critically ill patients and subsequent autopsy findings. Mayo Clinic Proceedings 75:562-567, 2000.

16. Rotimi 0 , Ajayi AA, Odesanmi WO. Sudden unexpected death from cardiac causes in nigerians: A review of 50 autopsied cases. International Journal of Cardiology 63:111-115, 1998 .

17. Vannucchi H, Cunha DF, Bernardes MM, Unamuno MRDL. Avaliação dos níveis séricos de vitaminas A, E, C e B , de carotenóides e zinco em idosos hospitalizados. Revista de Saúde Pública 28:121-126, 1994. 